\title{
Selective Determination of Serotonin on Poly(3,4-ethylenedioxy pyrrole)-single-walled Carbon Nanotube-Modified Glassy Carbon Electrodes
}

\author{
Seul Ki Kim, Si Ra Bae, Mohammad Shamsuddin Ahmed, Jung-Min You, and Seungwon Jeon* \\ Department of Chemistry and Institute of Basic Science, Chonnam National University, Gwangju 500-757, Korea \\ *E-mail: swjeon3380@naver.com \\ Received November 29, 2010, Accepted February 7, 2011
}

\begin{abstract}
An electrochemically-modified electrode [P(EDOP-SWNTs)/GCE] was prepared by electropolymerization of 3,4-ethylenedioxy pyrrole (EDOP) single-walled carbon nanotubes (SWNTs) on the surface of a glassy carbon electrode (GCE) and characterized by SEM, CV, and DPV. This modified electrode was employed as an electrochemical biosensor for the selective determination of serotonin concentrations at $\mathrm{pH} 7.4$ and exhibited a typical enhanced effect on the current response of serotonin with a lower oxidation overpotential. The linear response was in the range of $1.0 \times 10^{-7}$ to $1.0 \times 10^{-5} \mathrm{M}$, with a correlation coefficient of 0.998 on the anodic current. The lower detection limit was calculated as $5.0 \mathrm{nM}$. Due to the relatively low currents and difference of potentials in the electrochemical responses of uric acid (UA), ascorbic acid (AA), and dopamine (DA), the modified electrode was a useful and effective sensing device for the selective and sensitive serotonin determination in the presence of UA, AA, and DA.
\end{abstract}

Key Words : 3,4-Ethylenedioxy pyrrole, Single-walled carbon nanotubes, Serotonin, Modified electrode, Biosensor

\section{Introduction}

Serotonin (5-hydroxytryptamine, 5-HT) is an important biomolecule in physiological systems, a neurotransmitter widely dispersed throughout the central nervous system, playing a vital role in the regulation of mood, sleep, emesis, sexuality, and appetite. Low levels of 5-HT are associated with several disorders, including depression, anxiety, and migraines. ${ }^{1,2}$ Extremely high levels of 5-HT can manifest toxicity and potentially fatal effects known as serotonin syndrome. ${ }^{3}$ It is well known that 5-HT and DA influence each other in their respective releasing given their coexistence in biological systems. ${ }^{4}$ Determination of 5 -HT is often complicated by the presence of DA, with the oxidation potential of 5-HT $(0.38 \mathrm{~V})$ close to that of DA $(0.22 \mathrm{~V})$. Furthermore, accurate determination of 5-HT often proves difficult because of interference from AA and UA, as both are often in high concentration and capable of oxidation at a potential close to that of 5-HT at the bare electrode. Several methods have been employed for accurate determination of 5-HT, such as liquid chromatography, fluorometry, coulometry, capillary electrophoresis, mass spectrometry, and electrochemical methods. ${ }^{5-15}$ With the exception of electrochemical methods, there exists several difficulties that render the methods overly time-consuming, such as high cost and requisite sample manipulations. Conducting polymers such as polypyrrole and polyaniline possess many attractive features as molecular recognition systems. Such polymers can be used to construct solid-state ion selective electrodes ${ }^{16-20}$ and composites with various nanomaterials and nanotubes, ${ }^{21-28}$ imparting superior selectivity and sensitivity toward neurotransmitter analytes. ${ }^{4}$ Research in the field of voltammetric sensors based on polymers are focused on three areas: (a) greater reproducibility in the formation of the electrode surface; (b) stable polymerizations that enables an acceptable durability of the sensors; (c) increase in the electrode surface in order to obtain greater levels of sensitivity. Additionally, the importance of the 5-HT level in usual matters requires a simple cost effective method for its rapid monitoring. Electroanalytical methods meet the above requirements, but normally possess lower sensitivity than conventional electrodes. However, low sensitivity and poor selectivity of electrochemical methods can be overcome through electrode modification. Nanogold, carbon nanotubes (CNTs) and fullerene-modified electrodes have been used by several research groups for simple, fast, and sensitive voltammetric determination of various compounds of physiological importance; ${ }^{29-33}$ an enhanced sensitivity has been observed with shifts of the peak potential to less positive potentials. Carbon nanotubes have stimulated increasing interest in the application of fabricated electrochemical sensors due to their unique combination of excellent mechanical, electrical, and electrochemical properties. ${ }^{34-36}$ Many applications of CNTs in electrochemical sensors for different biomolecules have been reported. ${ }^{37,38}$ In recent years, CNT-modified electrodes made using electropolymerization as a binder for analytical purposes have been reported in many studies; this gives new capabilities to electrochemical sensors by combining the advantages of both a copolymer and CNTs. Due to their attractive performance, CNT-copolymer composite materials have been utilized to fabricate electrochemical sensors for selective detection of various biomolecules. ${ }^{39}$

In this study, a poly(EDOP-SWNTs)-modified GCE was prepared by electrochemical polymerization via a cyclic 
voltammetry method. This modified electrode was employed for electrochemical selective determination of 5-HT in the comparable presence of DA, UA, and excess AA, with a linear response range of $1.0 \times 10^{-7}$ to $1.0 \times 10^{-5} \mathrm{M}$, under a biologically and analytically significant $\mathrm{pH}$ of 7.4; this was comparable to recent literature values. ${ }^{4,5,40}$ The respective detection limit and correlation coefficient were calculated as $5.0 \mathrm{nM}$ and 0.998 on the anodic current.

\section{Experimental}

Chemicals. The EDOP, tetrabutyl ammonium perchlorate (TBAP), 5-HT, DA, UA, and AA were purchased from Aldrich. The SWNTs [diam. $\times$ length: $1.2-1.5 \mathrm{~nm} \times 2.0-5.0$ $\mu \mathrm{m}$ (bundles), produced by an arc method, purity $50-70 \%$ ] were also purchased from Aldrich. All other reagents used were of analytical grade and used without further purification. All electrochemical experiments were carried out at room temperature. The $\mathrm{pH}$ of the PBS was adjusted with $0.1 \mathrm{M} \mathrm{H}_{3} \mathrm{PO}_{4}$ and $0.1 \mathrm{M} \mathrm{NaOH}$. High purity argon was used for deaeration. Doubly distilled water with a resistivity exceeding $18 \mathrm{M} \Omega \mathrm{cm}$ in a quartz apparatus was used to prepare all aqueous electrolyte solutions. Testing solutions of 5-HT, DA, UA, and AA were prepared by adding required volumes of the respective stock PBS solution.

Apparatus. A three-electrode assembled cell was employed, which consisted of the modified glassy carbon electrode $(3.0 \mathrm{~mm}$ diameter) as the working electrode. A platinum-wire electrode was used as an auxiliary electrode. An $\mathrm{Ag} / \mathrm{AgCl}(3.0 \mathrm{M} \mathrm{NaCl})$ electrode supplied by BAS (Model MF-2052) was used as the reference electrode. All potentials were reported with respect to the $\mathrm{Ag} / \mathrm{AgCl}$ electrode at room temperature and under an argon atmosphere. Electrochemical techniques, including cyclic voltammetry (CV), differential pulse voltammetry (DPV), and chronoamperometry (CA) were performed using a BAS 100B/W voltammetric analyzer (Bioanalytical Systems, West Lafayette, IN, USA) in a grounded Faraday cage. All pH measurements were performed with a $\mathrm{pH}$ glass electrode with a JENCO meter. Field emission scanning electron microscopic (FESEM) images of the modified electrode were obtained by a JSM-600 field emission scanning electron microanalyzer

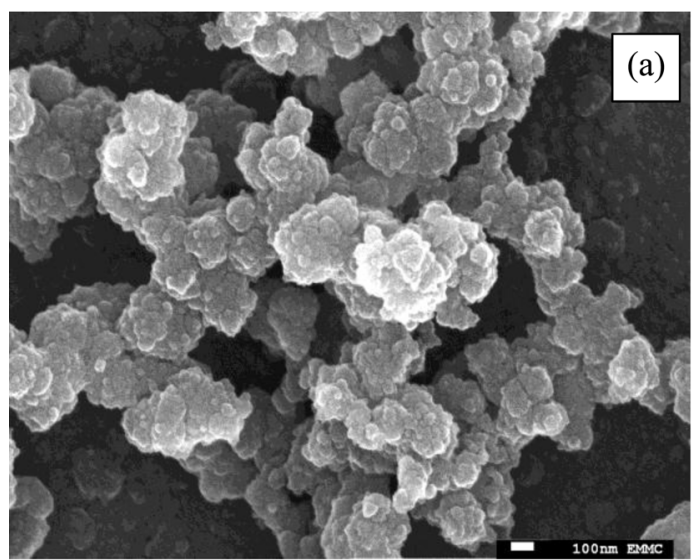

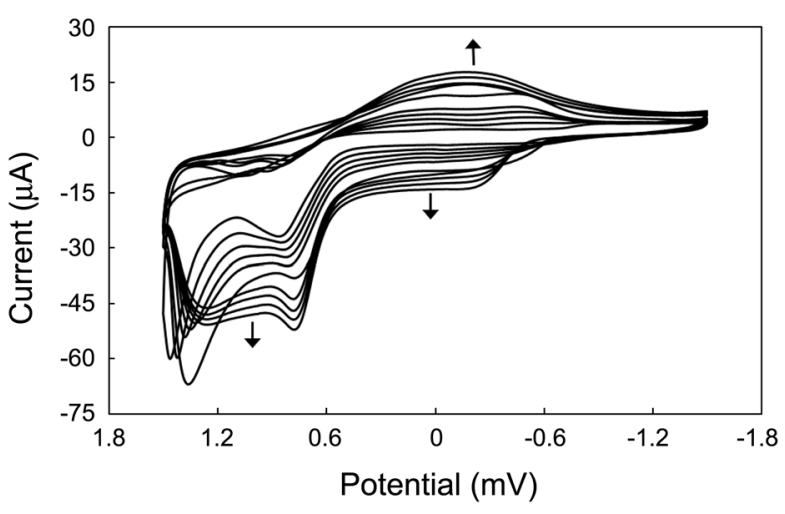

Figure 1. CVs of the electropolymerization of EDOP in the presence of SWNTs on GCE; Scan rate of $0.1 \mathrm{~V} / \mathrm{s}$ for 10 cycles.

\section{(JEOL).}

Preparation of the Modified Electrode. The GCE surface was highly polished with alumina paste, followed with a wash in a $1.0 \mathrm{M} \mathrm{HCl}$ solution, rinsed several times with distilled water, and finally rinsed with methanol. First, 1.0 $\mathrm{mM}$ EDOP, $1.0 \mathrm{mg}$ of SWNTs, and $0.05 \mathrm{M}$ TBAP/MeCN solution was mixed by ultrasonication for $5 \mathrm{~min}$; the P(EDOPSWNTs)-modified GCE was then made by electropolymerization with EDOP in the prepared solution with a cyclic sweeping from 1.5 to $-1.5 \mathrm{~V}$ at $0.1 \mathrm{~V} \mathrm{~s}^{-1}$ for 10 cycles (Fig. 1 ).

Also, the SWNTs/GCE surface was coated with $5.0 \mu \mathrm{L}$ of the black SWNT suspension $(1.0 \mathrm{mg} / \mathrm{mL})$ in distilled water. The solvent was evaporated in air at room temperature. The modified electrode was washed with distilled water before and after each experiment. All experiments for determination of 5-HT were carried out in a $15 \mathrm{~mL}$ electrolytic cell with $5.0 \mathrm{~mL}$ PBS, whereby oxygen was removed by a continuous purging with high-purity argon.

\section{Results and Discussion}

Electropolymerization of EDOP-SWNTs on GCE. The P(EDOP-SWNTs)-modified GCE was made by electropolymerization of $1.0 \mathrm{mM}$ EDOP and $1.0 \mathrm{mg}$ SWNTs in $0.05 \mathrm{M}$ TBAP/MeCN, with a cyclic sweeping as outlined in Figure 1. The reduction peak at $-0.2 \mathrm{~V}$ and the oxidation peaks at -0.2 and $+0.7 \mathrm{~V}$ were increased with subsequent cyclic

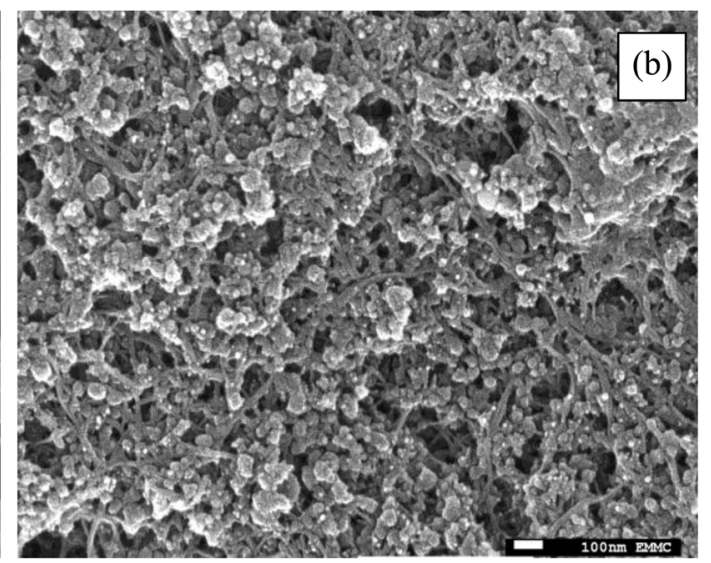

Figure 2. SEM image of electropolymerized P(EDOP)/GCE (a); P(EDOP-SWNTs)/GCE (b), in 0.05 M TBAP/MeCN. 
sweeping; this oxidation and reduction wave increased due to formation of a conducting polymer layer around the electrode, provoking broadening of the electronic surface.

Surface Morphology of the P(EDOP-SWNTs)/GCE. The SEM image of the surface of the modified GCE is shown in Figure 2. After electropolymerization of EDOP and (EDOP-SWNTs), the GCE bore variations in their structures, clearly indicating the existence of polymeric EDOP on the SWNTs. In Fig. 2(a), the EDOP was polymerized on the GCE surface in a crystalline form, though not completely homogeneous. Nevertheless, the EDOP was polymerized with SWNTs as a net-like structure and was homogeneously compact (Fig. 2(b)). The image illustrates the uniform dispersion of the poly (EDOP-SWNTs) on the GCE surface.

Electrochemical Behavior of 5-HT at the Modified Electrode. The voltammetric response of 5-HT on the modified electrode was performed via $\mathrm{CV}$. Figure 3 demonstrates a typical CV of $3.0 \times 10^{-6} \mathrm{M} 5-\mathrm{HT}$ in PBS at $\mathrm{pH} 7.4$, recorded at: bare GCE (a); completely modified electrodes (b, absence of 5-HT and c, presence of 5-HT); partially modified electrodes ( $\mathrm{d}$ and $\mathrm{e}$ ). At the bare electrode, a weak oxidation peak was observed near $0.39 \mathrm{~V}$, as shown in Figure 3(a). At the partially modified electrode were shallow (low current response) peaks at 0.34 and $0.39 \mathrm{~V}$, as shown in Figure 3(d) and 3(e), respectively. Under the same conditions, the anodic peak of $3.0 \times 10^{-6} \mathrm{M} 5$-HT at the completely modified electrode P(EDOP-SWNTs)/GCE appeared as a deep peak at $0.37 \mathrm{~V}$; the peak current increased significantly (Fig. 3c) and there was no signal at P(EDOP-SWNTs)/GCE due to absence of 5-HT (Fig. 3b). The remarkable enhancement in the current response of the oxidation potential provided clear evidence of the catalytic effects of the modified electrode acting as a promoter to enhance the electrochemical reaction and thus, considerably accelerating the rate of electron transfer. The scan rate was $0.1 \mathrm{~V} \mathrm{~s}^{-1}$, with the accumulation time of only $10 \mathrm{~s}$.

Electrochemical Response of 5-HT at the Modified Electrode. DPVs of 5-HT at the P(EDOP-SWNTs)/GCE (Fig. 4) showed a dynamic response due to increased con-

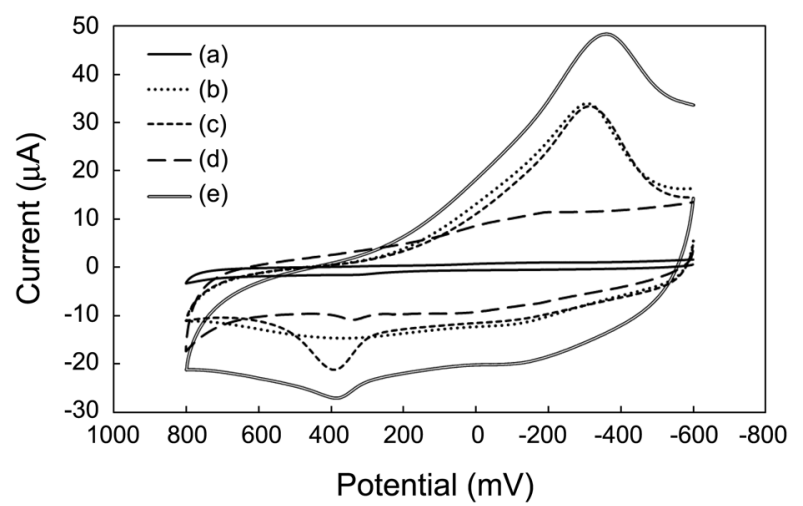

Figure 3. CVs at the bare GCE (a); P(EDOP-SWNTs)/GCE (b) and (c); SWNTs/GCE (d); P(EDOP)/GCE (e), each in PBS at pH 7.4 ; all electrodes were tested in the presence of $3.0 \times 10^{-6} \mathrm{M} 5$-HT, except (b).

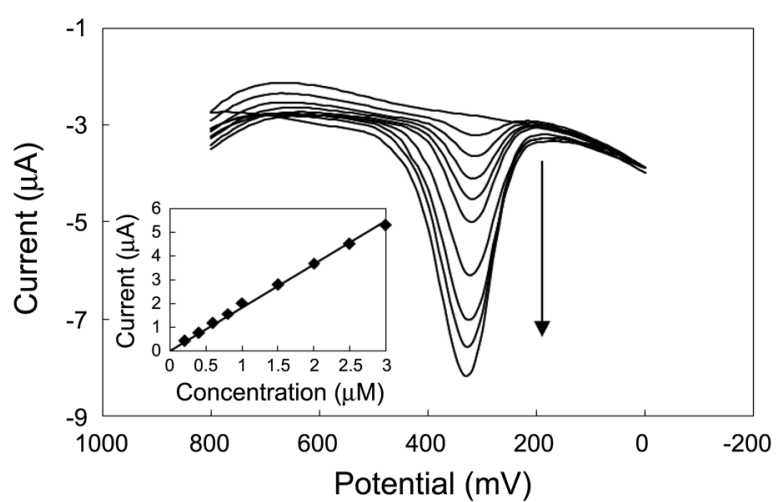

Figure 4. DPVs of 0.0 to $3.0 \times 10^{-6} \mathrm{M} 5-\mathrm{HT}$ at the P(EDOPSWNTs)/GCE in PBS, the plot of peak current versus 5-HT concentration (Inset).

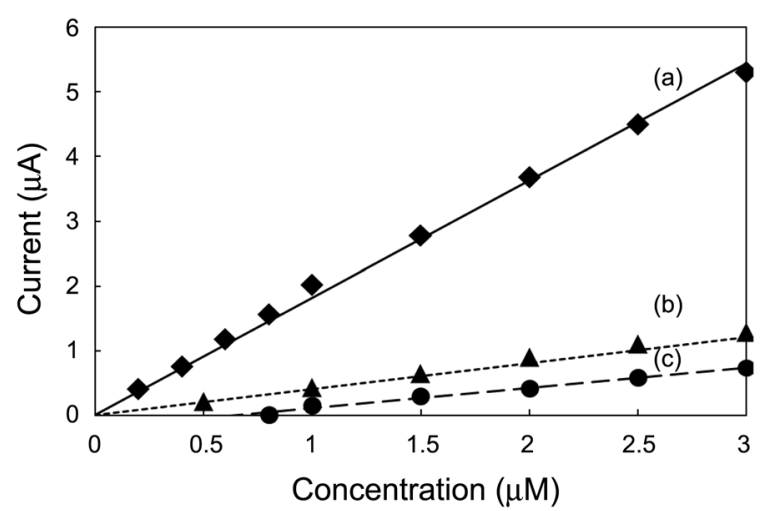

Figure 5. Plot of peak current versus concentration of 5-HT (a); DA (b); UA (c), all obtained from DPVs.

centration $\left(0.0\right.$ to $\left.3.0 \times 10^{-6} \mathrm{M}\right)$ of 5-HT; the sharp peak was found at $0.34 \mathrm{~V}$. The calibration curve for the 5-HT was measured using DPV. The catalytic peak current was found to increase as the concentration of 5-HT increased linearly (Fig. 4, inset). For 5-HT, the plot of the peak current $(\mu \mathrm{A})$ versus concentration (comparison with DA and UA, Fig. 5a) was linear in the range of $1.0 \times 10^{-7}$ to $3.0 \times 10^{-6} \mathrm{M}$, at $\mathrm{pH}$ 7.4. The linearity of the plot was reasonable, with a correlation coefficient of $0.996\left[i_{\mathrm{p}}(\mu \mathrm{A})=1.816 \mathrm{C}(\mu \mathrm{M})\right]$ on the anodic current, indicating that 5-HT could be estimated in the given concentration range. The voltammetric behaviors of UA, DA, and 5-HT mixture were investigated by DPV. While the plot of the peak current $(\mu \mathrm{A})$ for DA (Fig. 5(b), $0.990)$ and UA (Fig. 5(c), 0.988) versus concentration was also linear, it still could not provide interference based upon 5-HT determination. On the basis of the slope (Fig. 5), the electrochemical response of the 5-HT was significantly larger than that of DA and UA.

Furthermore, Figure 6 illustrates the chronoamperometric response of the modified electrode to subsequent additions of 5-HT in PBS at an applied potential of $0.38 \mathrm{~V}$, using CA. The inset of Figure 6 represents the plot of the response current versus 5-HT concentration. The linearity of the plot proved very reasonable. The linear regression equation is: $i(\mathrm{nA})=0.214 \mathrm{C}(\mathrm{nM})$, with a correlation coefficient of 0.998 . 


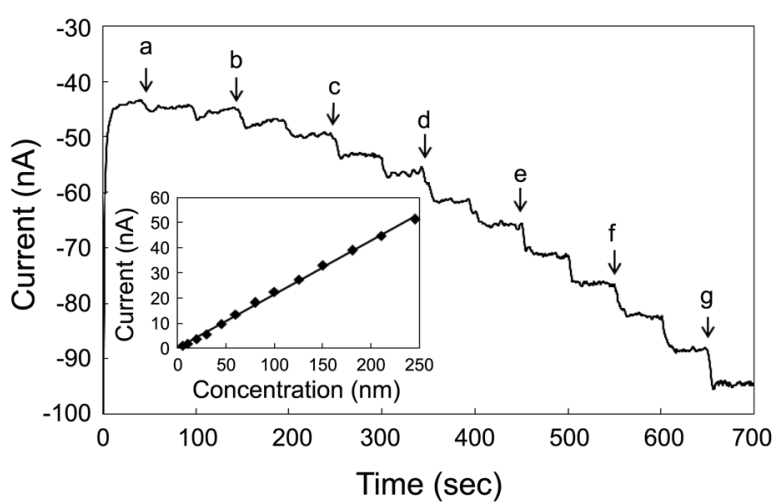

Figure 6. Amperometric response of 5-HT at the P(EDOP-SWNTs) /GCE in PBS at a constant potential of $380 \mathrm{mV}$. $\left(\mathrm{a} \rightarrow \mathrm{b}, 5.0 \times 10^{-9}\right.$ $\mathrm{M} ; \mathrm{b} \rightarrow \mathrm{c}, 1.0 \times 10^{-8} \mathrm{M} ; \mathrm{c} \rightarrow \mathrm{d}, 1.5 \times 10^{-8} \mathrm{M} ; \mathrm{d} \rightarrow \mathrm{e}, 2.0 \times 10^{-8} \mathrm{M}$; $\mathrm{e} \rightarrow \mathrm{f}, 2.5 \times 10^{-8} \mathrm{M} ; \mathrm{f} \rightarrow \mathrm{g}, 3.0 \times 10^{-8} \mathrm{M} ; \mathrm{g} \rightarrow, 3.5 \times 10^{-8} \mathrm{M}$ step). Inset: plot of response currents versus 5 -HT concentration.

This result suggests that 5-HT could be easily detected at very low concentrations using the chronoamperometric method, indicating a detection limit $(\mathrm{S} / \mathrm{N}=3)$ of $5.0 \mathrm{nM}$.

Differential pulse voltammetry was used for determination of 5-HT in the presence of AA and UA at the P(EDOPSWNTs)-modified GCE, given its higher sensitivity and improved resolution over $\mathrm{CV}$. As the aforementioned results indicated, the electrochemical response of 5-HT was stable in the successive DPV scan, with the well-separated oxidation peak of the 5-HT. Thus, a DPV scan for one cycle from 0 to $0.8 \mathrm{~V}$ or -0.1 to $0.8 \mathrm{~V}$ at $50 \mathrm{mV} / \mathrm{s}$ was performed with the mixture sample, containing different concentrations of 5-HT, DA, AA, and UA. However, as shown in Figure 7, in the presence of a constant concentration $\left(5.0 \times 10^{-6} \mathrm{M}\right)$ of UA, DPV was performed at different concentrations ( 0.0 to $3.0 \times$ $10^{-6} \mathrm{M}$ ) of 5-HT, yielding a peak near $500 \mathrm{mV}$ for UA. Accordingly, there was a peak near $0.34 \mathrm{~V}$ for 5 -HT that increased (current response) upon increase in 5-HT concentration. The peak was still in the previous position $(0.34 \mathrm{~V})$, indicating that the UA could not interfere with 5-HT electrolytic determination. No interference, even at 10 -fold UA, affected determination of the 5-HT level; a 10-fold peak current intensity of UA proved less than 5-HT.

To investigate the electrochemical response when 5 -HT

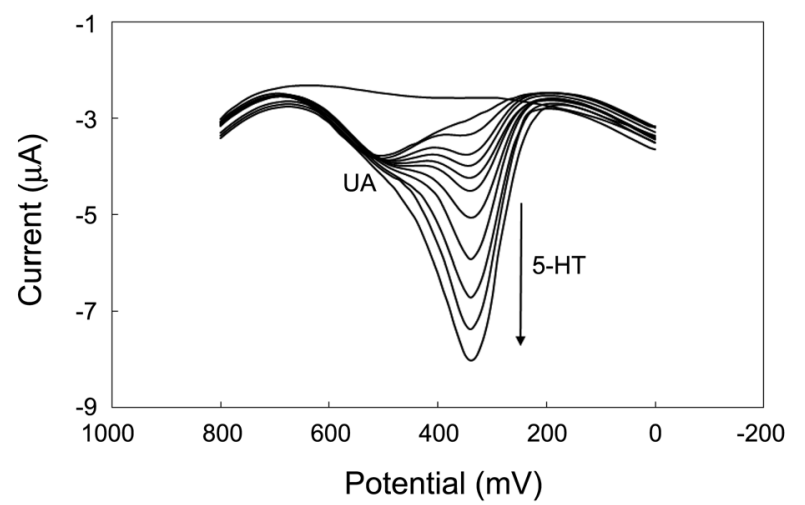

Figure 7. DPVs of 0.0 to $3.0 \times 10^{-6} \mathrm{M} 5$-HT in the presence of 5.0 $\times 10^{-6} \mathrm{M}$ UA at the P(EDOP-SWNTs)/GCE at $\mathrm{pH} 7.4$ in PBS.

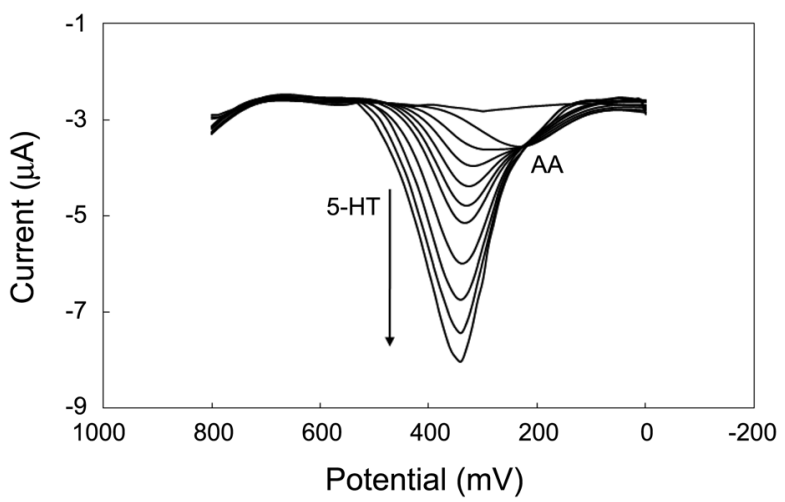

Figure 8. DPVs of 0.0 to $3.0 \times 10^{-6} \mathrm{M} 5-\mathrm{HT}$ in the presence of 5.0 $\times 10^{-4} \mathrm{M}$ AA at the P(EDOP-SWNTs)/GCE at pH 7.4 in PBS.

co-existed with AA, its voltammetric behavior was examined by DPV in the presence of a substantial excess of AA. All experiments were carried out by varying concentration $(0.0$ to $3.0 \times 10^{-6} \mathrm{M}$ ) of the 5 -HT in the presence of $5.0 \times 10^{-4} \mathrm{M}$ (166-fold) AA (Fig. 8). Acceptable linearity was obtained between the anodic DPV peak currents of the 5-HT concentration in the mixture. Clearly, the competitive adsorption equilibrium of 5-HT could be overcome at a suitable concentration. Hence, this proposed method was suitable for concentration determination of 5-HT, since the amount of AA in the biological system was larger than the monoamine neurotransmitters. Interference of the high concentration of AA should be taken into account prior to determination of 5HT. From the above discussion, it is apparent that AA does not interfere with measurement of 5-HT at pH 7.4 in PBS, at a scan rate of $50 \mathrm{mV} / \mathrm{s}$, and the accumulation time of $10 \mathrm{~s}$.

Figure 5 represents the variation in peak current observed at different concentrations of 5-HT, DA, and UA. The $i_{\mathrm{pa}}$ was found to increase linearly with concentration, with a correlation coefficient of 0.990 on the anodic current of DA and within a measuring range of $1.0 \times 10^{-7}$ to $3.0 \times 10^{-6} \mathrm{M}$ for 5-HT. On the basis of the slope, the electrochemical response of 5-HT was significantly larger than that of DA and UA at the P(EDOP-SWNTs)/GCE. A possible reason for such a response can be that oxidation of the analyte was subject to substrate modification on the GCE or morphology of the modified electrode. Until now, the modified electrodes demonstrated the electrochemical response of 5-HT to be smaller than that of DA. ${ }^{4,40}$ These results suggest that, in the presence of DA, the concentrations of 5-HT can be selectively determined with excellent sensitivity. It is possible that the amino group of the 5-HT is more electroactive than that of DA, according to structure. The oxidative electrochemistry of 5-HT is, however, complex. In Figure 9, different concentrations $\left(0.0\right.$ to $\left.3.0 \times 10^{-6} \mathrm{M}\right)$ of 5 -HT were measured with $1.0 \times 10^{-5} \mathrm{M} \mathrm{DA}$, without any interference with the determination of 5-HT. Under identical conditions, DA and 5-HT yielded two well-defined oxidation peaks with respective potentials of 0.18 and $0.34 \mathrm{~V}$ at the P(EDOP-SWNTs) film-coated GCE; they did not interfered with each other. Consequently, the electron transfers of DA and 5-HT at the 


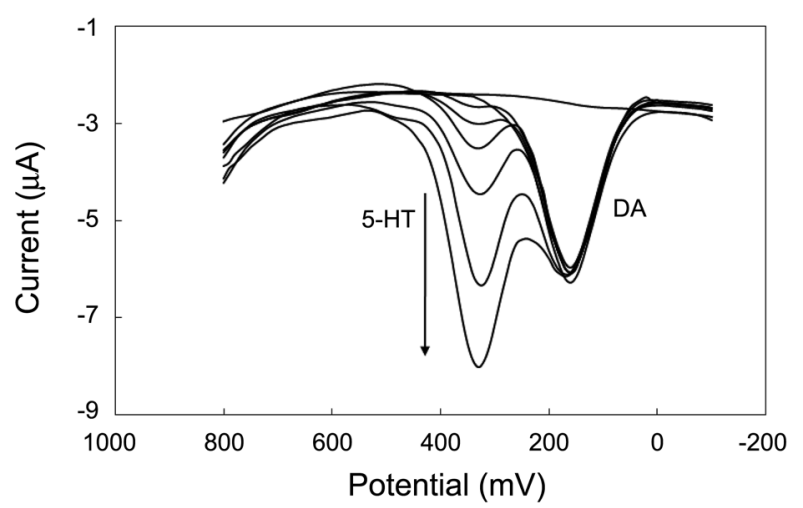

Figure 9. DPVs of 0.0 to $3.0 \times 10^{-6} \mathrm{M} 5$-HT in the presence of 1.0 $\times 10^{-5} \mathrm{M}$ DA at the P(EDOP-SWNTs)/GCE in PBS.

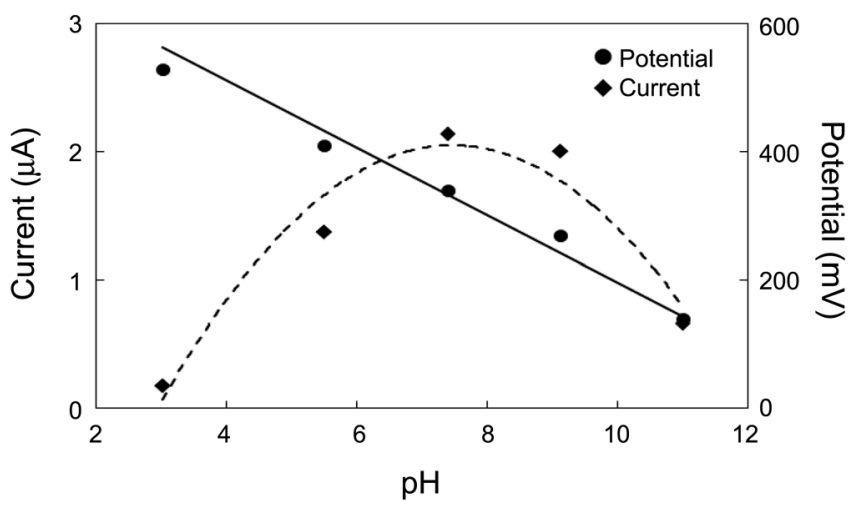

Figure 10. Effect of $\mathrm{pH}$ on $1.0 \times 10^{-6} \mathrm{M} 5$-HT oxidation at the modified electrode by $\mathrm{CV}$.

$\mathrm{P}($ EDOP-SWNTs)/GCE were facilitated. Thus, DA and 5HT yielded two separate and sensitive oxidation peaks.

Effect of pH on P(EDOP-SWNTs)/GCE. The effect of $\mathrm{pH}$ on the electrode response for the oxidation of 5-HT was investigated using the $\mathrm{CV}$ mode in a $\mathrm{pH}$ range $3-11$, and in the presence of $1.0 \times 10^{-6} \mathrm{M} 5$-HT. The peak currents of the 5 -HT increased with an increase in $\mathrm{pH}$, indicating the proportion of the electrons and protons involved in the oxidation of 5-HT. Finally, the highest oxidation peak currents were observed at pH 7.4 PBS, as shown in Figure 10. The physiological $\mathrm{pH}$ of 7.4 in PBS was chosen as the supporting electrolyte for the experiment. The slope for the 5 -HT was $-52.55 \mathrm{mV} / \mathrm{pH}$, which was in agreement for the $2 \mathrm{e}^{-}$transfer reaction. ${ }^{41}$

Effect of Scan Rate and Adsorption of 5-HT on P(EDOP-SWNTs)/GCE. The influence of the scan rate on the oxidation peak current of 5-HT at the P(EDOP-SWNTs)/ GCE was also studied via $\mathrm{CV}$ in the presence of $2.0 \times 10^{-6}$ M 5-HT. As demonstrated in Figure 11(a), there were a wide-ranging linear peak currents of the 5-HT and a square root of the scan rate from 25 to $400 \mathrm{mV} / \mathrm{s}$, a linear regression equation of: $I_{\mathrm{pa}}(\mu \mathrm{A})=1.10 \mathrm{~V}^{1 / 2}-3.83$, and a correlation coefficient of 0.997 , indicating a diffusion-controlled process in the electrochemical reaction.

Under a different accumulation time, responses of 5-HT $\left(3.0 \times 10^{-6} \mathrm{M}\right)$ at $\mathrm{P}(\mathrm{EDOP}-\mathrm{SWNTs}) / \mathrm{GCE}$ were also investi-
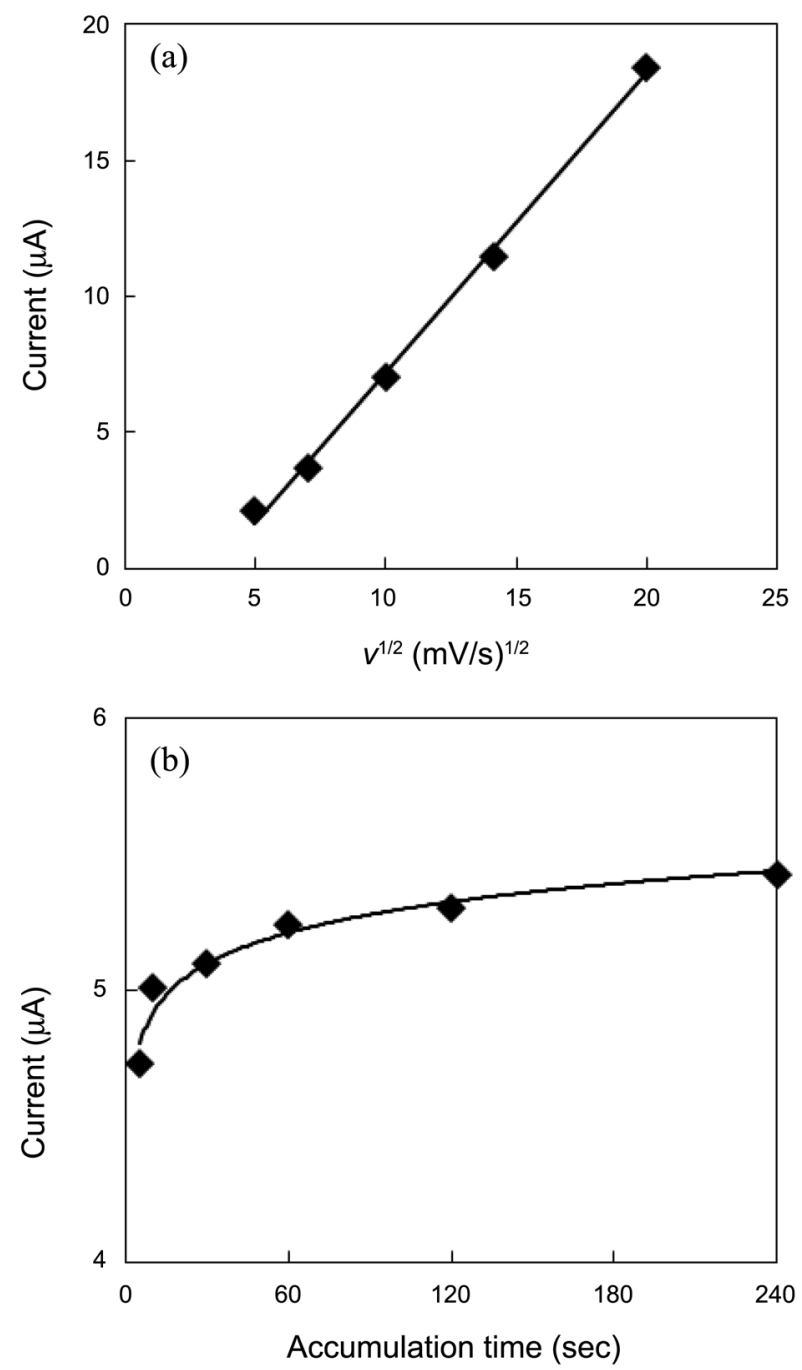

Figure 11. Effect of the scan rate of $3.0 \times 10^{-6} \mathrm{M}$ (a); adsorption of $2.0 \times 10^{-6} \mathrm{M} 5$-HT (b) on P(EDOP-SWNTs)/GCE by CV, investigated in PBS at $\mathrm{pH}$ 7.4.

gated. As shown in Figure 11(b), if the accumulation time was longer, the serotonin peak currents increased minimally from $10 \mathrm{~s}(5.006 \mu \mathrm{A})$ and $240 \mathrm{~s}(5.423 \mu \mathrm{A})$. However, the oxidation of serotonin is known to produce hydroxylated products, dimers, and other species that can irreversibly adsorb to the electrode, thus fouling the surface. ${ }^{7}$ Ten seconds of accumulation time proved sufficient to obtain a good current response for serotonin with the P(EDOPSWNTs)/GCE. Thus, this short accumulation time showed reduced fouling and increased reproducibility. Accordingly, the accumulation time chosen was $10 \mathrm{~s}$.

Stability. The limit of detection (defined as the concentration leading to a signal three times the standard deviation of the baseline noise) was $5.0 \mathrm{nM}$. To characterize the reproducibility of the modified electrode, repetitive measurements were carried out in $1.0 \times 10^{-6} \mathrm{M}$ 5-HT. The results from five successive measurements demonstrated a $1.01 \%$ relative standard deviation (RSD), suggesting good reproducibility. The long-term stability of the modified electrode was estimated by measuring the current response at a fixed 
concentration of $1.0 \times 10^{-6} \mathrm{M} 5$-HT, over a period of $30 \mathrm{~d}$, with the modified electrode undergoing daily use. The experimental results showed that the current response deviated only $4.2 \%$, implying that the P(EDOP-SWNTs)/GCE possesses long-term stability. As a result, it could be stored in PBS ( $\mathrm{pH}$ 7.4) at approximately $20^{\circ} \mathrm{C}$.

\section{Conclusions}

The P(EDOP-SWNTs)-coated GCE exhibited outstanding electrocatalytic effects towards the oxidation of 5-HT, improving oxidation peak currents and lowering oxidation overpotential. Electrocatalysis by the P(EDOP-SWNTs)/ GCE toward 5-HT was mainly attributed to a stereo-porous interfacial layer. The single-walled carbon nanotubes provide many active sites, enhancing the sensitivity of 5-HT. At the bare GCE, the oxidation signal of the 5-HT merged and could not be sensitively determined. However, the P(EDOPSWNTs)/GCE permitted a favorable voltammetric resolution of 5-HT in the pH 7.4 phosphate buffer. The modified electrode demonstrated efficacy in detecting 5-HT selectively and exhibited excellent stability and reproducibility. Furthermore, a large excess of AA and UA had minimal influence over the oxidation signal of 5-HT. Thus, 5-HT could be easily and selectively oxidized in the presence of a certain amount of DA. These properties indicate the P(EDOP$\mathrm{SWNTs}$ )/GCE as promising for in vitro 5-HT measurements.

Acknowledgments. This research was supported by Basic Science Research Program through the National Research Foundation of Korea (NRF) funded by the Ministry of Education, Science and Technology (KRF-2008-313-C00571).

\section{References}

1. Lin, M. T.; Tsay, H. J.; Su, W. H.; Chueh, F. Y. Am. J. Physiol.Regul. 1998, 274, 1260.

2. Imeri, L.; Mancia, M.; Bianchi, S.; Opp, M. R. Neuroscience 2000, 95,445 .

3. Isbister, G. K.; Bowe, S. J.; Dawson, A.; Whyte, I. M. Clin. Toxicol. 2004, 42, 277.

4. Li, J.; Lin, X. Sens. Actuator B-Chem. 2007, 24, 486.

5. Yao, H.; Li, S.; Tang, Y.; Chen, Y.; Lin, X. Electrochim. Acta 2009, 54, 4607.

6. Selvaraju, T.; Ramaraj, R. Electrochem. Commun. 2003, 5, 667.

7. Swamy, B. E. K.; Venton, B. J. Analyst 2007, 132, 876.

8. Peterson, Z. D.; Collins, D. C.; Bowerbank, C. R.; Lee, M. L.; Graves, S. W. J. Chromatogr. B 2002, 776, 221.

9. Lema, M.; Otero, J.; Marco, J. J. Chromatogr. A 1991, 47, 113.
10. Cheng, F. C.; Shih, Y.; Liang, Y. J.; Yang, L. L.; Yang, C. S. J. Chromatogr. B 1996, 682, 195.

11. Bielavska, M.; Kassa, J. Collect. Czech. Chem. Commun. 2000 , 65, 1677.

12. Hsieh, M. M.; Chang, H. T. Electrophoresis 2005, 26, 187.

13. Yoshitake, T.; Kehr, J.; Todoroki, K.; Nohta, H.; Yamaguchi, M. Biomed. Chromatogr. 2006, 20, 267.

14. Baranowska, I.; Zydron, M. J. Planar Chromatogr.-Mod. TLC 2003, 6, 102.

15. Li, Y.; huang, X.; chen, Y.; Wang, L.; Lin, X. Microchim. Acta 2009, 64, 107

16. Migdilski, J.; Blaz, T.; Lewenstam, A. Anal. Chim. Acta 1996, $322,141$.

17. Cadogan, A.; Lewenstam, A.; Ivaska, A. Talanta 1992, 39, 617.

18. Cadogan, A.; Gao, Z. Q.; Lewenstam, A.; Ivaska, A.; Diamond, D. Anal. Chem. 1992, 64, 2469.

19. Weidlich, C.; Mangold, K. M.; Juttner, K. Electrochim. Acta 2001, $47,741$.

20. Weidlich, C.; Mangold, K. M.; Juttner, K. Electrochim. Acta 2005 , $50,5247$.

21. Cheng, D. M.; Xia, H. B.; Chan, H. S. O. Langmuir 2004, 20, 9909.

22. Lui, Y. C.; Lee, H. T.; Yang, S. T. Electrochim. Acta 2006, 51, 3441.

23. Zhang, P.; Yangb, Z. H.; Wang, D. J.; Kan, S. H.; Chai, X. D.; Liu, J. Z.; Li, T. J. Synth. Met 1997, 84, 165.

24. Li, Y.; Shi, G. Q. J. Phys. Chem. B 2005, 109(50), 23787.

25. Cioffi, N.; Torsi, L.; Losito, I.; Franco, C. D.; Bari, I. D.; Chiavarone, L.; Scamarcio, G.; Tsakova, V.; Sabbatini, L.; Zambonin, P. G. J. Mater. Chem. 2001, 11, 1434

26. Dahman, Y. J. Nanosci. Nanotechnol. 2009, 9, 5105.

27. Baron, R.; Wildgoose, G. G.; Compton, R. G. J. Nanosci. Nanotechnol. 2009, 9, 2274.

28. Kumar, R.; Cronin, S. B. J. Nanosci. Nanotechnol. 2008, 8, 122.

29. Wu, K.; Fei, J.; Hu, S. Anal. Biochem. 2003, 318, 100.

30. Goyal, R. N.; Oyama, M.; Gupta, V. K.; Singh, S. P.; Sharma, R. A. Sens. Actuator B-Chem. 2008, 134, 816.

31. Goyal, R. N.; Oyama, M.; Sangal, A.; Singh, S. P. Indian J. Chem. 2005, 44A, 945 .

32. Goyal, R. N.; Singh, S. P. Talanta 2006, 69, 932.

33. Goyal, R. N.; Oyama, M.; Singh, S. P. J. Electroanal. Chem. 2007, $611,140$.

34. Balasubramanian, K.; Burghard, M. Anal. Bioanal. Chem. 2006, $385,452$.

35. Trojanowicz, M. Trac-Trends Anal. Chem. 2006, 25, 480.

36. Pumera, M.; Eanchez, S.; Ichinose, I.; Tang, J. Sens. Actuator BChem. 2007, 123, 1195 .

37. Vamvakaki, V.; Chaniotakis, N. A. Sens. Actuator B-Chem. 2007, 126, 193.

38. Britto, P. J.; Santhanam, K. S. V.; Ajayan, P. M. Bioelectrochemistry 1996, 41, 121.

39. Jacobs, C. B.; Peairs, M. J.; Venton, B. J. Anal. Chim. Acta 2010, 662, 105.

40. Sun, Y.; Fei, J.; Hou, J.; Zhang, Q.; Liu, Y.; Hu, B. Microchim. Acta 2009, 165, 373.

41. Lin, X.; Zhang, Y.; Chen, W.; Wu, P. Sens. Actuator B-Chem. 2007, 122, 309. 